Volume 141, Number 12, December 2013, Pages 4345-4348

S 0002-9939(2013)11709-0

Article electronically published on August 28, 2013

\title{
A REMARK ON INVARIANT SUBSPACES OF POSITIVE OPERATORS
}

\author{
VLADIMIR G. TROITSKY
}

(Communicated by Thomas Schlumprecht)

\begin{abstract}
If $S, T, R$, and $K$ are non-zero positive operators on a Banach lattice such that $S \leftrightarrow T \leftrightarrow R \leqslant K$, where " $\leftrightarrow$ " stands for the commutation relation, $T$ is non-scalar, and $K$ is compact, then $S$ has an invariant subspace.
\end{abstract}

Throughout this note, $X$ is a (real or complex) Banach lattice. For two operators $S$ and $T$ on $X$, the notation $S \leftrightarrow T$ means that $S$ and $T$ commute. A (norm closed) subspace $Y$ of $X$ is said to be invariant under an operator $T$ in $L(X)$ if $\{0\} \neq Y \neq X$ and $T Y \subseteq Y$. We follow the notation and terminology of AA02.

There have been many extensions of Lomonosov's theorem Lom73 to positive operators; see Chapter 10 of AA02 for a review of the subject. In particular, if $T \leftrightarrow$ $R \geqslant K$ for some positive non-zero operators $T, R$, and $K$ with $T$ quasinilpotent and $K$ compact, then $T$ has an invariant subspace (even an invariant closed ideal). The condition $T \leftrightarrow R \geqslant K$ can be replaced with $T \leftrightarrow R \leqslant K$ or, even more generally, with $T \leftrightarrow R \geqslant C \leqslant K$ for some non-zero positive operator $C$; in the latter case, $T$ is said to be compact friendly. There have been several more recent similar extensions of Lomonosov's theorem to positive quasinilpotent operators: Drn01, IM04, AT05, ÇE07, FTT08, PT09, Ges09, FV09, DK11. In this note we do not require that $T$ be quasinilpotent. Our result was motivated by Theorem 3.5 of ÇM11, where quasinilpotence is not required either.

Theorem 1. Suppose that $S, T, R$, and $K$ are non-zero positive operators on a Banach lattice such that $S \leftrightarrow T \leftrightarrow R \leqslant K, T$ is non-scalar, and $K$ is compact. Then $S$ has an invariant subspace.

Proof. Suppose that $S$ has no invariant subspaces. Let $\widetilde{S}=\sum_{n=0}^{\infty} t^{n} S^{n}$, where $t$ is a positive real such that series converges. Then $\widetilde{S} \geqslant I, \widetilde{S} \geqslant t S$, and $\widetilde{S}$ commutes with $T$.

Claim. For every $x>0$, the vector $\widetilde{S} x$ is quasi-interior; that is, the order ideal $J$ generated by $\widetilde{S} x$ is dense in $X$. Indeed, $\widetilde{S} x \geqslant x>0$, so that $J \neq\{0\}$. Note that $J$ is invariant under $S$ because for every $z \in J$ we have $|z| \leqslant \lambda \widetilde{S} x$ for some $\lambda>0$,

Received by the editors December 1, 2011 and, in revised form, February 8, 2012 and February $19,2012$.

2010 Mathematics Subject Classification. Primary 47B65; Secondary 47A15.

Key words and phrases. Positive operators, invariant subspaces.

The author was supported by NSERC. 
so that

$$
|S z| \leqslant S|z| \leqslant \lambda S \widetilde{S} x=\lambda \sum_{n=0}^{\infty} t^{n} S^{n+1} x=\frac{\lambda}{t} \sum_{n=0}^{\infty} t^{n+1} S^{n+1} x \leqslant \frac{\lambda}{t} \widetilde{S} x .
$$

Since $S$ has no invariant subspaces, $J$ has to be dense in $X$. This proves the claim.

Since $R \neq 0$, there exists $x_{0}>0$ such that $R x_{0}>0$. By the claim, $\widetilde{S} R x_{0}$ is quasi-interior. Since $R$ is positive and non-zero, it cannot vanish on a quasi-interior vector; hence $R \widetilde{S} R x_{0}>0$. Iterating this step, we get $R \widetilde{S} R \widetilde{S} R x_{0}>0$. It follows that $R \widetilde{S} R \widetilde{S} R \neq 0$. Since $\widetilde{S} R \leqslant \widetilde{S} K$ and the latter operator is compact, $R \widetilde{S} R \widetilde{S} R$ is compact by Aliprantis-Burkinshaw's Cube Theorem [AA02, Theorem 2.34]. Hence, $T$ commutes with a non-zero compact operator. Therefore, $T$ has a hyperinvariant subspace: in case of a complex Banach lattice this follows from Lomonosov's Theorem, while in the case of a real Banach lattice we use Corollary 2.4 of [Sir05].

Remark 2. We have, actually, proved more than stated: we proved that either $S$ has an invariant closed ideal or $T$ commutes with a non-zero compact operator and, therefore, has a hyperinvariant subspace. We would also like to point out that the assumption that $T$ is positive is not really needed.

To put Theorem 1 in perspective, note that under the assumptions of the theorem, the following facts are well known:

- If both $X$ and $X^{*}$ have order continuous norm, then $R$ is compact by the Dodds-Fremlin Theorem [AA02, Theorem 2.38], so that $T$ has a hyperinvariant subspace by Lomonosov's Theorem.

- Note that $R^{3}$ is always compact by the Cube Theorem and $T \leftrightarrow R^{3}$. Thus, if $R^{3} \neq 0$, then it follows immediately from Lomonosov's Theorem that $T$ has a hyperinvariant subspace. On the other hand, if $R^{3}=0$, then ker $R$ is a non-trivial subspace invariant under $T$. Hence, in any case, $T$ has an invariant subspace.

- Note that $T$ is compact-friendly. Therefore, if $T$ is quasinilpotent at a positive vector, then Theorem 10.55 of [AA02 guarantees that $S$ has an invariant closed ideal. The following result is an analogue of Theorem 10.55 in our setting.

Theorem 3. Suppose that $T, R$, and $K$ are non-zero positive operators on a $B a$ nach lattice $X$ such that $T \leftrightarrow R \leqslant K, T$ is non-scalar, and $K$ is compact. If $\left(S_{n}\right)$ is a sequence of positive operators commuting with $T$, then there is a subspace invariant under $T, R$, and all $S_{n}$ 's.

Proof. Let $S=T+R+\sum_{n=1}^{\infty} a_{n} S_{n}$, where $\left(a_{n}\right)$ is a sequence of positive reals such that the series converges. Observe that $S$ is a positive operator commuting with $T$. If $S$ has an invariant closed ideal, then this ideal remains invariant under $T, R$, and each $S_{n}$ because these operators are dominated by $S$. However, if $S$ has no invariant closed subspaces, then $T$ has a hyperinvariant subspace by Remark 2 .

Example 4. $0 \leqslant R \leqslant K, K$ is compact, $R$ is not compact, and $R^{2}=0$.

This is the case in Example 5.19 of [AB06]; it is one of the few classical examples showing that the Dodds-Fremlin Theorem may fail when $X^{*}$ is not order continuous. Here is the example. Put $X=\ell_{1} \oplus L_{2}$. Let $\left(e_{i}\right)_{i=1}^{\infty}$ stand for the unit vector basis 
of $\ell_{1},\left(r_{i}\right)_{i=1}^{\infty}$ stand for the sequence of the Rademacher functions in $L_{2}$, and $r_{0}=\mathbb{1}$ stand for the constant one function in $L_{2}$. Recall that the sequence $\left(r_{i}\right)_{i=0}^{\infty}$ is an orthonormal sequence in $L_{2}$. Note also that $r_{i}^{+}=\frac{1}{2}\left(r_{i}+\mathbb{1}\right)$ for all $i$. We define $R_{0}, K_{0}: \ell_{1} \rightarrow L_{2}$ via $K_{0} e_{i}=\mathbb{1}$ and $R_{0} e_{i}=r_{i}^{+}$for all $i \geqslant 1$. It is easy to see that both operators are bounded, $K_{0}$ is compact, $R_{0}$ is not compact, and $0 \leqslant R_{0} \leqslant K_{0}$. Now put $R=\left[\begin{array}{cc}0 & 0 \\ R_{0} & 0\end{array}\right]$ and $K=\left[\begin{array}{cc}0 & 0 \\ K_{0} & 0\end{array}\right]$. Then $R$ and $K$ are two operators on $X$ with $0 \leqslant R \leqslant K, K$ is compact, $R$ is not compact, and $R^{2}=0$.

Example 5. With $R$ and $K$ as in Example 4, we will construct $T$ such that $T$ commutes with $R$ but not with $K$. Put $T=\left[\begin{array}{ll}P & 0 \\ 0 & Q\end{array}\right]$, where $P: \ell_{1} \rightarrow \ell_{1}$ is the left shift: $P e_{i}=e_{i-1}$ if $i>1$ and $P e_{1}=0$, and $Q: L_{2} \rightarrow L_{2}$ is defined as follows. Put $Q \mathbb{1}=\mathbb{1}, Q r_{1}=-\mathbb{1}, Q r_{i}=r_{i-1}$ for $i>1$ and define $Q$ arbitrarily on the orthogonal complement of the closed span of $\left(r_{i}\right)_{i=0}^{\infty}$ in $L_{2}$. Using the fact that $r_{i}^{+}=\frac{1}{2}\left(r_{i}+\mathbb{1}\right)$ we see that $Q$ acts as a left shift on the sequence $\left(r_{i}^{+}\right)_{i=1}^{\infty}$. It is easy to see that $T$ commutes with $R$ because for every $\sum_{i=1}^{\infty} \alpha_{i} e_{i}$ in $\ell_{1}$ and every $f \in L_{2}$ we have $T R\left(\sum_{i=1}^{\infty} \alpha_{i} e_{i}, f\right)=\left(0, \sum_{i=1}^{\infty} \alpha_{i+1} r_{i}^{+}\right)=R T\left(\sum_{i=1}^{\infty} \alpha_{i} e_{i}, f\right)$. However, $T$ does not commute with $K$ because $T K\left(e_{1}, 0\right)=(0, \mathbb{1})$, while $K T\left(e_{1}, 0\right)=(0,0)$. Note that $T$ is not positive.

Example 6. We construct three non-zero positive operators $T, R$, and $K$ such that $0 \leqslant R \leqslant K, K$ is compact, $R$ is not compact, and $T$ commutes with $R$ but not with $K$. In particular, the operators $K, R$, and $T$, together with any positive operator $S$ which commutes with $T$, satisfy the assumptions of Theorem 1 .

We construct $R$ and $K$ similarly to Example 4. We again put $X=\ell_{1} \oplus L_{2}$, but this time we consider $\ell_{1}$ indexed by $\mathbb{N} \cup\{0\}$, so that the unit basis now starts with $e_{0}$. Again, we define $R=\left[\begin{array}{cc}0 & 0 \\ R_{0} & 0\end{array}\right]$ and $K=\left[\begin{array}{cc}0 & 0 \\ K_{0} & 0\end{array}\right]$, where $R_{0} e_{i}=r_{i}^{+}$and $K_{0} e_{i}=\mathbb{1}$ for all $i=0,1,2, \ldots$ (recall that $r_{0}=\mathbb{1}$ ). We still have $0 \leqslant R \leqslant K, K$ is compact, $R$ is not compact, and $R^{2}=0$. Put $T=\left[\begin{array}{ll}P & 0 \\ 0 & Q\end{array}\right]$, where $P: \ell_{1} \rightarrow \ell_{1}$ and $Q: L_{2} \rightarrow L_{2}$ are defined as follows. Fix a positive real parameter $\alpha$. For $f \in L_{2}$, put

$$
(Q f)(t)=f\left(\frac{t}{2}\right)+2 \alpha \int_{\frac{1}{2}}^{1} f, \quad t \in[0,1] .
$$

It is easy to see that $Q \mathbb{1}=(1+\alpha) \mathbb{1}, Q r_{1}=(1-\alpha) \mathbb{1}$, and $Q r_{i}=r_{i-1}$ for $i>1$. It follows from $r_{i}^{+}=\frac{1}{2}\left(r_{i}+\mathbb{1}\right)$ that $Q r_{1}^{+}=\mathbb{1}$ and $Q r_{i}^{+}=r_{i-1}^{+}+\frac{\alpha}{2} \mathbb{1}$ whenever $i>1$. Now we define $P$ so that the action of $P$ on $\left(e_{i}\right)_{i=0}^{\infty}$ matches the action of $Q$ on $\left(r_{i}^{+}\right)_{i=0}^{\infty}$, namely,

$$
P e_{i}= \begin{cases}(1+\alpha) e_{0} & i=0 \\ e_{0} & i=1 \\ e_{i-1}+\frac{\alpha}{2} e_{0} & i>1\end{cases}
$$

Clearly, $Q$ and $P$ are positive; hence so is $T$. It is easy to verify that $T$ commutes with $R$. However, $T$ does not commute with $K$ as $T K\left(e_{1}, 0\right)=T(0, \mathbb{1})=(0$, $(1+\alpha) \mathbb{1})$, while $K T\left(e_{1}, 0\right)=K\left(e_{0}, 0\right)=(0, \mathbb{1})$.

Note that $(0, \mathbb{1})$ is an eigenvector of $T$; it follows that $T$ has a hyperinvariant subspace. Also, if $\alpha=1$, then $T$ commutes with the compact positive operator $C$ defined by $C(x, f)=\left(0,\left(\int_{0}^{1} f\right) \mathbb{1}\right)$. We do not know whether $T$ commutes with a compact operator when $\alpha \neq 1$. 


\section{REFERENCES}

[AA02] Y. A. Abramovich and C. D. Aliprantis, An invitation to operator theory, Graduate Studies in Mathematics, vol. 50, American Mathematical Society, Providence, RI, 2002. MR1921782(2003h:47072)

[AAB93] Y. A. Abramovich, C. D. Aliprantis, and O. Burkinshaw, Invariant subspaces of operators on $l_{p}$-spaces, J. Funct. Anal. 115 (1993), no. 2, 418-424, DOI 10.1006/jfan.1993.1097. MR,1234398 (94h:47009)

[AAB94] Y. A. Abramovich, C. D. Aliprantis, and O. Burkinshaw, Invariant subspace theorems for positive operators, J. Funct. Anal. 124 (1994), no. 1, 95-111, DOI 10.1006/jfan.1994.1099. MR1284604 (95e:47006)

[AB06] Charalambos D. Aliprantis and Owen Burkinshaw, Positive operators, Springer, Dordrecht, 2006. Reprint of the 1985 original. MR2262133

[AT05] Razvan Anisca and Vladimir G. Troitsky, Minimal vectors of positive operators, Indiana Univ. Math. J. 54 (2005), no. 3, 861-872, DOI 10.1512/iumj.2005.54.2544. MR2151236 (2006c:47041)

[ÇE07] M. Çağlar and Z. Ercan, Invariant subspaces for positive operators on locally convex solid Riesz spaces, Indag. Math. (N.S.) 18 (2007), no. 3, 417-420, DOI 10.1016/S00193577(07)80030-9. MR2373689(2008k:47012)

[ÇM11] Mert Çă̆lar and Tunç Misırlığlu, A note on a problem of Abramovich, Aliprantis and Burkinshaw, Positivity 15 (2011), no. 3, 473-480, DOI 10.1007/s11117-010-0096-2. $\operatorname{MR} 2832600$

[DK11] Roman Drnovšek and Marko Kandić, More on positive commutators, J. Math. Anal. Appl. 373 (2011), no. 2, 580-584, DOI 10.1016/j.jmaa.2010.07.056. MR2720706 (2011j:47123)

[Drn01] Roman Drnovšek, Common invariant subspaces for collections of operators, Integral Equations Operator Theory 39 (2001), no. 3, 253-266, DOI 10.1007/BF01332655. MR $1818060(2001 \mathrm{~m}: 47012)$

[FTT08] Julio Flores, Pedro Tradacete, and Vladimir G. Troitsky, Invariant subspaces of positive strictly singular operators on Banach lattices, J. Math. Anal. Appl. 343 (2008), no. 2, 743-751, DOI 10.1016/j.jmaa.2008.01.067. MR2401530(2009c:47008)

[FV09] A. Fernández Valles, Invariant ideals for uniform joint locally quasinilpotent operators, Rocky Mountain J. Math. 39 (2009), no. 5, 1699-1712, DOI 10.1216/RMJ-2009-39-51699. MR2546660 (2010i:47064)

[Ges09] Hailegebriel E. Gessesse, Invariant subspaces of super left-commutants, Proc. Amer. Math. Soc. 137 (2009), no. 4, 1357-1361, DOI 10.1090/S0002-9939-08-09673-1. MR2465659 (2009i:47017)

[IM04] Maria Cristina Isidori and Anna Martellotti, Invariant subspaces for compactfriendly operators in Sobolev spaces, Positivity 8 (2004), no. 2, 109-122, DOI 10.1023/B:POST.0000042839.07106.d6. MR2097082 (2005m:47083)

[Lom73] V. I. Lomonosov, Invariant subspaces of the family of operators that commute with a completely continuous operator, Funkcional. Anal. i Priložen. 7 (1973), no. 3, 55-56 (Russian). MR0420305 (54 \#8319)

[PT09] Alexey I. Popov and Vladimir G. Troitsky, A version of Lomonosov's theorem for collections of positive operators, Proc. Amer. Math. Soc. 137 (2009), no. 5, 1793-1800, DOI 10.1090/S0002-9939-08-09775-X. MR2470839(2010j:47008)

[Sir05] Gleb Sirotkin, A version of the Lomonosov invariant subspace theorem for real Banach spaces, Indiana Univ. Math. J. 54 (2005), no. 1, 257-262, DOI 10.1512/iumj.2005.54.2561. MR2126724(2006f:47007)

Department of Mathematical and Statistical Sciences, University of Alberta, Edmonton, AB, T6G 2G1, CAnada

E-mail address: troitsky@ualberta.ca 